\title{
Phototriggered Control of Enzyme Reactions toward High-throughput Screening on a Microwell Array Chip
}

\author{
Shingo Ueno ${ }^{1,2^{*}}$ and Takanori Ichiki ${ }^{1,2}$ \\ ${ }^{1}$ Innovation Center of NanoMedicine, Institute of Industry Promotion-Kawasaki, \\ 3-25-14 Tonomachi, Kawasaki-ku, Kawasaki, 210-0821, Japan \\ 2 Department of Materials Engineering, School of Engineering, The University of Tokyo, \\ 7-3-1 Hongo, Bunkyo-ku, Tokyo 113-8656, Japan \\ *ueno-s@kawasaki-net.ne.jp
}

\begin{abstract}
The spatiotemporal control of enzyme reactions based on the combined use of a caged substrate and local photoirradiation was proposed for use in the high-throughput screening of enzymes on a microwell array chip. An enzyme reaction is initiated immediately after the mixing of an enzyme and its substrate. Since, rapid injection or addition of a reagent to initiate the reaction with appropriate timing is required for the fine measurement of the reaction. However, it is usually very difficult to inject an additional reagent in micrometer-sized wells. The coupled reactions of pyruvate kinase and luciferase with caged adenosine diphosphate (caged ADP) were used as a model of enzyme reactions to be controlled by phototriggering. The coupled reactions were suppressed until photoirradiation and were initiated by the photoirradiation at 340-380 $\mathrm{nm}$. The extent of the reactions was proved to be controlled by adjusting the photoirradiation dose; $500 \mathrm{~mJ} / \mathrm{cm}^{2}$ was sufficient to saturate the extent of the triggered reactions with $0.1 \mathrm{mM}$ caged ADP in $50 \mu \mathrm{L}$. Multistep measurements of enzyme reactions by reusing the same reaction solution were carried out by multistep phototriggering at fixed or various doses. The phototriggering technique enables the measurement of enzyme reactions in micrometer-sized microwells.
\end{abstract}

Keywords: Phototriggering, Caged molecule, Microarray, Microwell array, Enzyme screening, High-throughput screening

\section{Introduction}

Enzyme properties are conventionally improved or modified by the microtiter-plate-based screening of mutant enzymes, which are extracted from cultured bacteria or other microorganisms [1]. Indeed, microtiter-plate-based screening systems have achieved certain results, but their screening capacity, generally up to tens of thousands of library size, is insufficient for searching a novel enzyme variant from a huge and diverse library of mutant enzymes (e.g., regarding the possible diversity, a mutant library constructed by the randomization of three amino acids in an enzyme composed of 300 amino acids would include approximately $3.6 \times 10^{10}$ variants).

Recently, protein microarrays composed of miniaturized microwell reactors and in situ synthesized proteins have been developed for the high-throughput screening of proteins including enzymes [2-5]. Microwell-array-based highthroughput screening would be a powerful tool to improve or engineer enzymes. The miniaturization of microwells down to the order of micrometer with femtoliter volume has been proceeded to increase the throughput of screening and reduce the amount of reagents used.

Generally, triggering of the initiation of an enzyme reaction is required for its quantitative kinetic measurement. In microtiter-plate-based measurement, the triggering is done by injection of a reagent. However, in microwell-array-based measurement, the injection of a reagent to each microwell is technically difficult because of the smallness of the microwells. Therefore, there was an issue to be solved for quantitative kinetic measurement of an enzyme reaction using a 
microwell arrays chip.

A caged molecule is inactivated via a photoremovable protection group and activated by spatiotemporal photoirradiation for uncaging [6]. So far, caged compounds have been used as a means to control biochemical reactions spatiotemporally (i.e., localized neuronal stimulation $[7,8]$, conformational change of proteins [9-11], and gene expression [12-14]). Thus, the use of a caged molecule enables us to rapidly change the concentration of a molecule at any location and time, even in a closed space such as a cell, a droplet, or a sealed vessel.

In this article, a photocontrolling technique for enzyme reactions in microwells using a caged molecule is proposed. Figure 1 shows the concept of the photocontrolled measurement of enzyme reactions on an array of microwells by spatiotemporal photoirradiation. In the scheme, enzyme reactions can be measured in any well at any time while preventing the undesirable initiation of any reactions outside the measurement area and period.

\section{Experimental}

2.1. Phototriggering of coupled reactions of pyruvate kinase and luciferase

TM buffer $(50 \mu \mathrm{L} ; 50 \mathrm{mM}$ Tris-HCl, $\mathrm{pH} 7.5$, and $5 \mathrm{mM} \mathrm{MgSO} 4$ ) containing $0.05 \mathrm{mg} / \mathrm{mL}$ luciferase from Photimus pyralis (Sigma-Aldrich, Co., LLC.), $0.5 \mathrm{mM}$ D-luciferin (Wako Pure Chemical Industries, Ltd.), $10 \mu \mathrm{g} / \mathrm{mL}$ pyruvate kinase from the rabbit muscle (Sigma-Aldrich, Co., LLC.), $1 \mathrm{mM}$ phosphoenolpyruvic acid (Wako Pure Chemical Industries, Ltd.), and $0.1 \mathrm{mM} \mathrm{P}{ }^{3}$-[1-(2-nitrophenyl) ethyl] adenosine-5'-diphosphate (caged ADP) was prepared in a microtiter plate (Corning Inc., Cat. \#3694). The luminescence of the luciferase reaction was measured at $30^{\circ} \mathrm{C}$ for 14 min using a Glomax 96 microplate luminometer (Promega Corp.). After the measurement, the plate was removed from the luminometer and the solution was irradiated with 0 $1500 \mathrm{~mJ} / \mathrm{cm}^{2} \mathrm{UV}$ light from a $250 \mathrm{~W}$ mercury lamp using Spot Cure SP-9 (Ushio Inc.) via a 340/20 nm band-pass filter (Nikon Corp.). After the irradiation, the luminescence of the solution was measured using the luminometer.

2.2. Multistep phototriggering of coupled reactions of pyruvate kinase and luciferase

TM buffer $(30 \mu \mathrm{L})$ containing $0.05 \mathrm{mg} / \mathrm{mL}$ luciferase, $0.5 \mathrm{mM}$ D-luciferin, $0.3 \mu \mathrm{g} / \mathrm{mL}$ pyruvate kinase, $1 \mathrm{mM}$ phosphoenolpyruvic acid, and 0.1

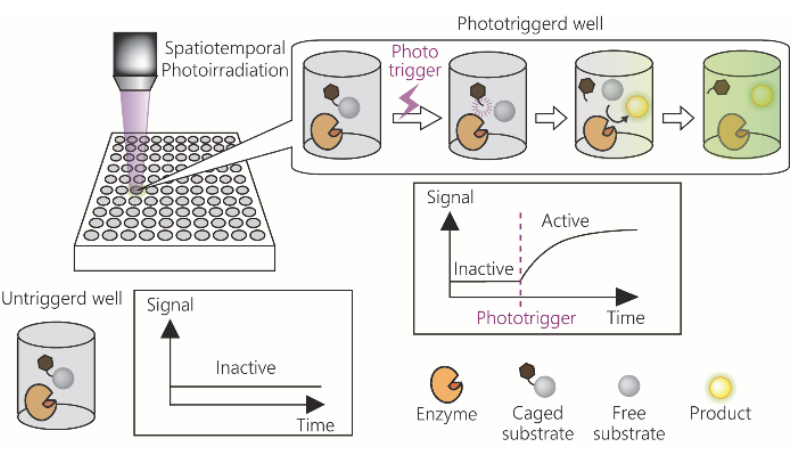

Fig. 1. Concept of phototriggering control of an enzyme reaction in a microwell array. The combination of the use of a caged substrate and spatiotemporal photoirradiation enables the trigger of the enzyme reaction in any well and at any time without the need to inject a reagent.

$\mathrm{mM}$ caged ADP was prepared in a microtiter plate. The luminescence of reactions was measured at $30^{\circ} \mathrm{C}$ using the luminometer. The plate was then removed from the luminometer and the solution was irradiated at a fixed dose $\left(100 \mathrm{~mJ} / \mathrm{cm}^{2}\right)$ or different doses $\left(0-100 \mathrm{~mJ} / \mathrm{cm}^{2}\right)$ of UV light via a $360 / 20 \mathrm{~nm}$ band-pass filter every $9 \mathrm{~min}$ during the measurement.

\section{Results and discussion}

3.1. Pyruvate kinase and luciferase coupled reactions

ATP regeneration systems using inexpensive materials are in demand for the industrial processes using ATP-dependent enzyme, because ATP is an expensive compound [15]. Pyruvate kinase regenerates ATP from ADP, which is a typical product of ATP-dependent enzyme reactions, via the transfer of a phosphate group from phosphoenolpyruvic acid to ADP [16].

A luciferase reaction is used for the detection and quantification of the activities of various enzymes by converting the enzymatic product into the luminescence of oxyluciferin $[17,18]$. A pyruvate kinase reaction can also be coupled to the luciferase reaction to detect the activity of pyruvate kinase via ATP, which is a product of the pyruvate kinase reaction and the substrate of the luciferase reaction, as shown below.

$$
\begin{aligned}
& \text { Phosphoenolpyruvate }+ \text { ADP } \\
& \underset{\text { Pyruvate kinase }}{ } \underset{\mathrm{Mg}^{2+}, \mathrm{K}^{+}}{ } \text {Pyruvate + ATP (1) }
\end{aligned}
$$




$$
\begin{gathered}
\text { Luciferin }+\mathrm{O}_{2}+\text { ATP } \frac{\text { Luciferase }}{\mathrm{Mg}^{2+}} \\
\text { Oxyluciferin }+\mathrm{hv}+\mathrm{AMP}+\mathrm{PPi}+\mathrm{CO}_{2}
\end{gathered}
$$

A

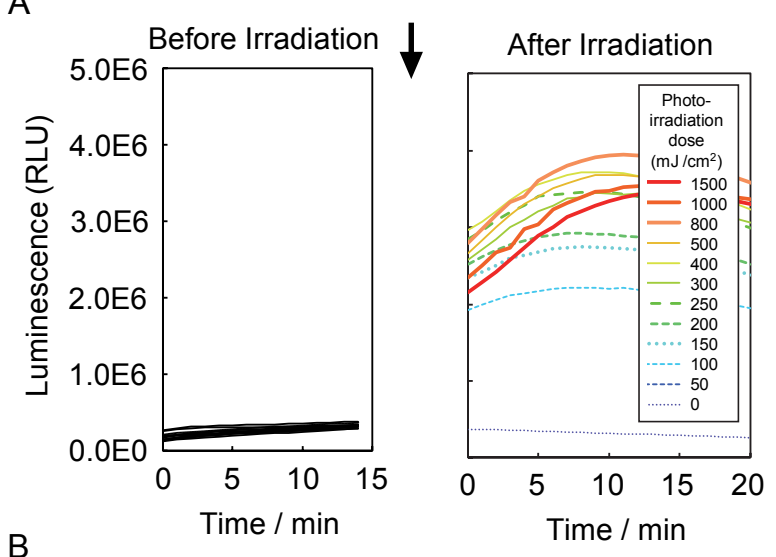

B

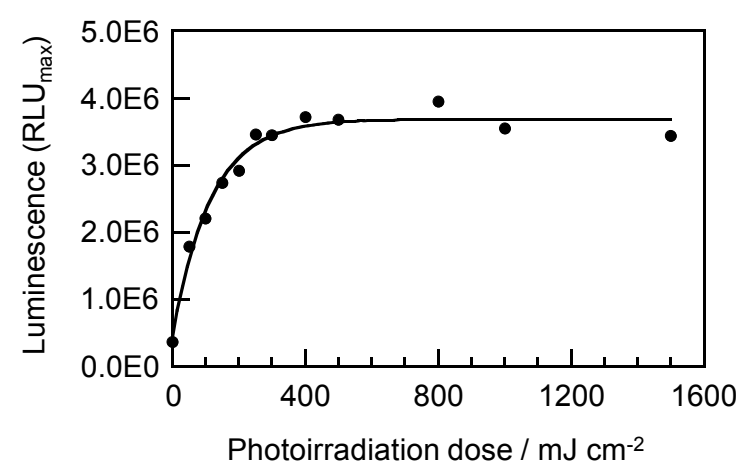

Fig. 2. (A) Phototriggering of coupled reaction of pyruvate kinase and luciferase with caged ADP. The time course of the luminescence intensity before and after the photoirradiation at different doses was measured. The intensity of the evoked luminescence was dependent on the dose of photoirradiation. The arrow indicates the time of photoirradiation. (B) Maximum luminescence intensity plotted as a function of the dose of photoirradiation. The solid line indicates the exponential fit.

The pyruvate kinase and luciferase coupled reactions with caged ADP instead of normal ADP can be controlled by photoirradiation, and detected by the luminescence generated from the coupled luciferase reactions. We used these coupled reactions as a model of photocontrolled enzyme reactions.

3.2. Phototriggering of pyruvate kinase and luciferase coupled reactions

The pyruvate kinase and luciferase coupled reactions with caged ADP were phototriggered. As shown in Fig. 2A, the reactions were suppressed until the time of photoirradiation and successfully initiated at the time of photoirradiation. The intensity of luminescence (i.e., the amount of the product of the coupled reactions) was dependent on the dose of photoirradiation and saturated at over $500 \mathrm{~mJ} / \mathrm{cm}^{2}$ under the conditions of this study (Fig. 2B). These results indicate that the extent of the reaction could be controlled by adjusting the photoirradiation dose.

\subsection{Multistep phototriggering of enzyme reaction}

As shown above, the extent of uncaging could be controlled by adjusting the photoirradiation dose. For the next step, multistep photoirradiations of the solution in the same well were performed as a feasibility study of multistep measurements of an enzyme reaction in a single well.

Successive irradiations at $100 \mathrm{~mJ} / \mathrm{cm}^{2}$ for $10 \mathrm{~min}$ each for the pyruvate kinase and luciferase coupled reaction with caged ADP were performed and the luminescence intensity of the reactions with time

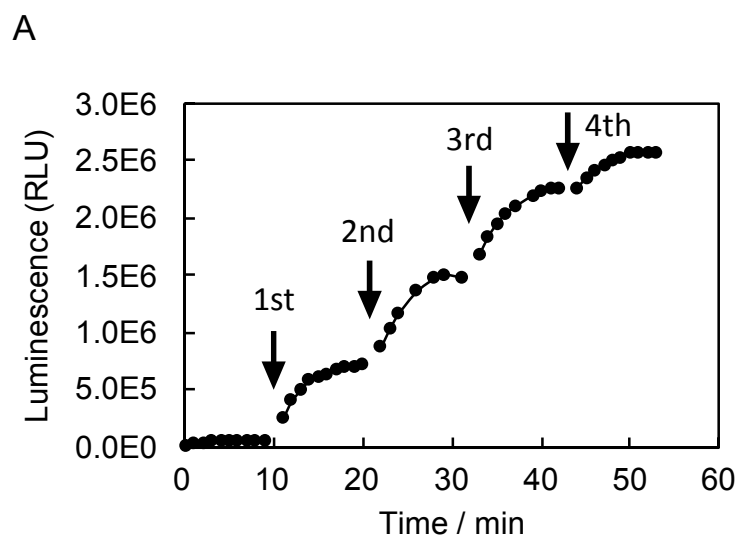

B

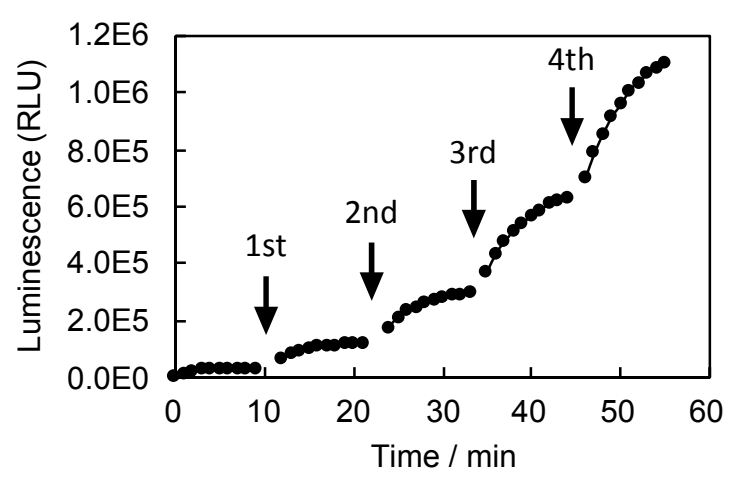

Fig. 3. Multistep phototriggering of the coupled reaction of pyruvate kinase and luciferase with caged ADP. The reaction in a single well was successively phototriggered at the same dose of photoirradiation $\left(100 \mathrm{~mJ} / \mathrm{cm}^{2}\right)(\mathrm{A})$, and the different doses of photoirradiation (1st, 25 $\mathrm{mJ} / \mathrm{cm}^{2} ; 2 \mathrm{nd}, 50 \mathrm{~mJ} / \mathrm{cm}^{2} ; 3 \mathrm{rd}, 75 \mathrm{~mJ} / \mathrm{cm}^{2}$; and 4 th, 100 $\mathrm{mJ} / \mathrm{cm}^{2}$ ) (B). Arrows indicate the time of photoirradiation. 
was measured. As a result, approximately the same increases in luminescence intensity were observed after each photoirradiation (Fig. 3A). This result indicates that multistep releases of a substrate of the same amount and multistep measurements of the reaction in the same well become possible by multistep phototriggering. This technique can be used to examine the properties of an enzyme in different environments using only a single well by multistep phototriggering under various environmental conditions.

In addition, multistep phototriggering at different photoirradiation doses were carried out. Figure 3B shows the results of the successive irradiations at different doses for $10 \mathrm{~min}$ each in the pyruvate kinase and luciferase coupled reactions with caged ADP. The different extents of increase in luminescence intensity depending on the photoirradiation doses were observed. This technique may be used to measure the reaction rate at different concentrations of a substrate in a single well to obtain the $V_{\max }$ and $K_{\mathrm{m}}$ of an enzyme using the Michaelis-Menten equation without the need to prepare reaction solutions with different concentrations of a substrate in multiple wells.

\section{Conclusions}

The phototriggered control of an enzyme reaction was carried out using the coupled reactions of pyruvate kinase and luciferase with caged ADP. The coupled reactions were initiated by the phototriggered release of ADP from caged ADP, and the progression of the initiated reaction was monitored by the coupled luciferase reaction. The photoirradiation dose of $500 \mathrm{~mJ} / \mathrm{cm}^{2}$ was sufficient to saturate the ADP release from $0.1 \mathrm{mM}$ caged ADP in a volume of $50 \mu \mathrm{L}$. The multistep initiations and measurements of the coupled reactions of pyruvate kinase and luciferase with caged ADP were demonstrated by multistep phototriggering of the same solution using a single well at the doses of 25 , 50,75 , and $100 \mathrm{~mJ} / \mathrm{cm}^{2}$ for $0.1 \mathrm{mM}$ caged ADP in a volume of $30 \mu \mathrm{L}$. The proof-of-concept of phototriggered control of an enzyme reaction was successfully demonstrated. This technique is expected to be applicable to the quantification of an enzyme reaction in a microwell in combination with the advancement in sensitive microscope-based assay technologies.

\section{Acknowledgements}

The authors thank Ms. Shioya for technical assistance. This work was supported by Research Complex Program of the Japan Science and Technology Agency (JST), and JSPS KAKENHI Grant Numbers JP15K13313, JP16K16644.

\section{References}

1. H. Leemhuis, R. M. Kelly, and L. Dijkhuizen, IUBMB Life, 61 (2009) 222.

2. N. Kilb, J. Burger, and G. Roth, Eng. Life Sci., 14 (2014) 352.

3. Y. Hosoi, T. Akagi, and T. Ichiki, Electron. Commun. Jpn., 92 (2009) 35.

4. S. Ueno, T. Hirai, S. Sato, M. Biyani, H. Kuramochi, R. Iizuka, T. Akagi, T. Funatsu, and T. Ichiki, J. Photopolym. Sci. Technol., 28 (2015) 719.

5. B. R. Takulapalli, J. Qiu, D. M. Maqee, P. Kahn, A. Brunner, K. Barker, S. Means, S. Miersch, X. Bian, A. Mendoza, F. Festa, K. Syal, J. G. Park, J. LaBaer, and P. Wiktor, J. Proteome Res., 11 (2012) 4382 .

6. G. C. Ellis-Davies, Nat. Methods, 4 (2007) 619.

7. R. H. Kramer, J. J. Chambers, and D. Trauner, Nat. Chem. Biol., 1 (2005) 360.

8. S. C. Rogan and B. L. Roth, Pharmacol. Rev., 63 (2011) 291.

9. T. Funatsu, E. Kono, and S. Tsukita, J. Cell Biol., 121 (1993) 1053.

10. H. Higuchi, E. Muto, Y. Inoue, and T. Yanagida, Proc. Natl. Acad. Sci. U S A, 94 (1997) 4395.

11. Y. Takei, R. Iizuka, T. Ueno, and T. Funatsu, $J$. Biol. Chem., 287 (2012) 41118.

12. W. T. Monroe, M. M. McQuain, M. S. Chang, J. S. Alexander, and F. R. Haselton, J. Biol. Chem., 274 (1999) 20895.

13. H. Ando, T. Furuta, R. Y. Tsien, and H. Okamoto, Nat. Genet., 28 (2001) 317.

14. T. Ohtsuki, S. Kanzaki, S. Nishimura, Y. Kunihiro, M. Sisido, and K. Watanabe, Nat. Commun., 7 (2016) 12501.

15. J. N. Andexer and M. Richter, ChemBioChem, 16 (2015) 380.

16. B. L. Hirschbein, F. P. Mazenod, and G. M. Whitesides, J. Org. Chem., 47 (1982) 3765.

17. F. Fan and K. V. Wood, Assay Drug Dev. Technol., 5 (2007) 127.

18. M. Ronaghi, S. Karamohamed, B. Pettersson, M. Uhlén, and P. Nyrén, Anal. Biochem., 242 (1996) 84. 